\title{
A library of $H$ band stellar spectra for stellar population analysis purposes $^{\star}$
}

\author{
R. Dallier, C. Boisson and M. Joly \\ DAEC, Observatoire de Paris, Université Paris VII, CNRS (UA 173), F-92195 Meudon Cedex, France
}

Received July 24; accepted September 25, 1995

\begin{abstract}
A sample of 37 stars of luminosity classes I, III, V, including few SMR stars observed in the $H$ band with a medium resolution $(R=1500-2000)$ is presented. This sample of flux calibrated spectra is intended to widen existing stellar libraries. A new IR spectrograph, ISIS/IR, foreseen for CFHT has been used. Its main characteristics are briefly described. Stellar spectra obtained with this new spectrograph are compared to similar data obtained with the ESO NTT IRSPEC spectrograph. The behaviour of some prominent features is described and it is shown that the $\operatorname{CO} \lambda 1.60 / \mathrm{SiI} \lambda 1.60$ line ratio is a good luminosity indicator for stellar types later than $\mathrm{K} 0$.
\end{abstract}

Key words: line: identification — stars: fundamental parameters: classification — stars: late type — Infrared: stars - atlases

\section{Introduction}

A very promising wavelength range not yet fully explored to study the stellar populations of the central part of galaxies is the near IR range where cool star spectrum peaks. The $H$ window is particularly well designed for such a study as the non-stellar contribution (mainly dust) is smaller in the $H$ window than in the $K$ window. For example, it has been shown by Origlia et al. (1993) that, in NGC 1068, the non-stellar contribution is only $30 \%$ in the $H$ window while it is about $80 \%$ in the $K$ window. Furthermore, these authors have shown that the line ratio $\mathrm{CO} \lambda 1.62 / \mathrm{SiI} \lambda 1.59$ increases strongly from early $\mathrm{K}$ to $\mathrm{M}$ stars and can be a crucial tool in the determination of the average spectral type in a stellar cluster.

As we are involved in the study of the stellar populations of active galactic nuclei, we need to compare the observed galactic spectra to stellar libraries of similar resolution. The compilation has to include the whole range of spectral types and luminosity classes.

Few stellar libraries are up to now available in the near IR. Each of them being constructed in a specific purpose, they are generally not exhaustive. Moreover some of them are restricted to the $K$ band (Arnaud et al. 1989; Bell et al. 1990; Kleinmann \& Hall 1986; see also, although less exhaustive, Casali \& Mathews 1992; Gaffney \& Lester 1992).

Send offprint requests to: $\mathrm{R}$. Dallier

${ }^{\star}$ Based on observations collected at the Canadian-FrenchHawaiian Telescope, Hawaii, and at the European Southern Observatory, La Silla, Chile
In the $H$ band, the more exhaustive library is by Lançon \& Rocca-Volmerange (1993) with 22 dwarf, 23 giant and 10 supergiant stars but with a poor spectral resolution $(R=500)$. Origlia et al. (1993), with a better resolution $(R=1500)$, present a sample of 13 supergiant stars of all types, 7 cool giant and 2 dwarf stars. Other authors restrict their study to K and M stars: Terndrup et al. (1991) study 32 giant $\mathrm{M}$ stars with a resolution $R=1000$; Davidge (1990) and Davidge \& Boeshaar (1993) study few K and $\mathrm{M}$ stars (giant and dwarf) with a low resolution $(R=300$ and 600). High resolution spectroscopy is also available in the near IR. Note for example the work of Mould (1978) who presents the spectra of six M dwarf stars in the $H$ and $K$ bands $\left(R=210^{4}\right)$, as well as the work of Tsuji (1986, $1991)$ on the first or second overtone of $\mathrm{CO}$ in cool giant stars $\left(R>5.10^{4}\right)$. Unfortunately, equivalent widths or line indices of prominent features are not always given by the authors and the spectra not easily available.

Very few super metal rich stars (SMR) are included in these stellar libraries (3 in Lançon \& Rocca-Volmerange 1993; and 5 in Origlia et al. 1993).

The inclusion of SMR stars in a stellar library dedicated to the study of stellar populations in the central part of galaxies is crucial as abundance gradients have been observed in the optical range with overabundances as high as a factor 5 compared to the solar vicinity (Cayrel de Strobel 1991; Vila-Costas \& Edmunds 1992; Davidge 1992; Delisle \& Hardy 1992).

In conclusion, we need to widen the previous data banks according to our purpose. We can use as a basis 
the stellar library from Origlia et al. (1993) which has a resolution close to the one we use for our galactic spectra $(R \simeq 1500-2000)$. It has to be extended with stellar spectra of different spectral types and luminosity classes including SMR stars.

We present here stellar spectra of intermediate resolution in the $H$ band, covering a large range of spectral types and luminosity classes, and including few SMR stars. These spectra have been mainly obtained with a new IR spectrograph which is quickly introduced in the next section. In Sect. 3 we describe the observations. The libraries are presented in Sect. 4 with comments on the behaviour of some prominent features.

Table 1.

\begin{tabular}{|c|c|c|c|c|c|c|}
\hline \multicolumn{2}{|c|}{ Star } & Type & | Tel. & \multicolumn{3}{|c|}{ | Spectro. | $[\mathrm{Fe} / \mathrm{H}] \mid$ Ref. } \\
\hline & $\begin{array}{l}\text { BS } 2456 \\
\text { BS } 1879 \\
\text { BS } 1123 \\
\text { BS } 5056 \\
\text { BS } 4590 \\
\text { BS } 4638 \\
\text { ISS } 4456 \\
\text { BS } 788 \\
\text { BS } 4983 \\
\text { HD } 160691 \\
\text { BS } 4550 \\
\text { H† } 112758 \\
\text { HD } 93800 \\
\text { HD } 130871 \\
\text { HD } 115404 \\
\text { HD } 157881 \\
\text { GL } 382\end{array}$ & $\begin{array}{l}O 7 \\
\text { OB } \\
\text { BoV } \\
\text { B1V } \\
\text { B } 1.5 \mathrm{~V} \\
\text { B.3V } \\
\text { B4V } \\
\text { FoV } \\
\text { GOV } \\
\text { G5V } \\
\text { G8V } \\
\text { KOV } \\
\text { KOV } \\
\\
\text { K2V } \\
\text { K2V } \\
\text { K7V } \\
\text { M2V }\end{array}$ & $\begin{array}{l}\text { CFHT } \\
\text { GFHT } \\
\text { CFHT } \\
\text { CFHT } \\
\text { CFHT } \\
\text { NTT } \\
\text { CFHT } \\
\text { CFHT } \\
\text { CFHT } \\
\text { NTT } \\
\text { CFHT } \\
\text { CFHT } \\
\text { NTT } \\
\text { CFHT } \\
\text { NTT } \\
\text { CFHT } \\
\text { NTT } \\
\text { NTT } \\
\text { CFHT }\end{array}$ & $\begin{array}{l}\text { ISIS/1R. } \\
\text { ISIS/IR } \\
\text { ISIS/IR } \\
\text { ISIS/IR } \\
\text { ISIS/IR } \\
\text { IRSPEC2 } \\
\text { ISIS/IR } \\
\text { ISIS/IR } \\
\text { ISIS/IR } \\
\text { IRSPEC2 } \\
\text { ISIS/IR } \\
\text { ISIS/IR. } \\
\text { IRSPEC2 } \\
\text { ISIS/IR } \\
\text { IRSPECZ } \\
\text { ISIS/IR } \\
\text { IRSPEC2 } \\
\text { IRSPEC2 } \\
\text { ISIS/IR }\end{array}$ & $\begin{array}{r}0.02 \\
0.16 \\
-1.40 \\
\\
0.43\end{array}$ & $\begin{array}{l}\text { C\&92 } \\
\text { C\&82 } \\
\text { C\&92 } \\
\text { BG90 }\end{array}$ \\
\hline & $\begin{array}{l}\text { BS } 1790 \\
\text { BS } 4883 \\
\text { BS } 3903 \\
\text { BS } 5020 \\
\text { BS } 4895 \\
\text { BS } 5315 \\
\text { BS } 5824 \\
\text { BS } 6973 \\
\text { HD } 111180 \\
\text { IID } 175674 \\
\text { BS } 2731 \\
\text { HD } 109655 \\
\text { BS } 4174 \\
\text { BS } 4127 \\
\text { BS } 4902\end{array}$ & $\begin{array}{l}\text { B2III } \\
\text { GOIII } \\
\text { G7III } \\
\text { G8III } \\
\text { KoIII } \\
\text { K3III } \\
\text { K3III } \\
\text { K3III } \\
\text { K3III } \\
\text { K3III } \\
\text { K5III } \\
\text { K5III } \\
\text { MOIII } \\
\text { MIIII } \\
\text { M3III }\end{array}$ & $\begin{array}{l}\text { CFHT } \\
\text { CFHT } \\
\text { CFHT } \\
\text { NT'T } \\
\text { CFHT } \\
\text { NTT } \\
\text { CFHT } \\
\text { CFHT } \\
\text { NTT } \\
\text { NTT } \\
\text { CFHT } \\
\text { NTT } \\
\text { CFHI } \\
\text { CFHT } \\
\text { NTT } \\
\text { CFHT } \\
\text { NTT } \\
\text { CFHT }\end{array}$ & \begin{tabular}{|l|} 
ISIS/IR \\
1SIS/IR \\
ISIS/IR \\
IRSPEC2 \\
ISIS/IR \\
IRSPEC2 \\
TSIS/IIR \\
ISIS/IR \\
IFASPE2 \\
IRSPEC2 \\
ISIS/IR \\
IRSPEC2 \\
ISIS/IR \\
ISIS/IR \\
IRSPEC2 \\
ISIS/IR \\
IISSPEC2 \\
ISIS/IF
\end{tabular} & $\begin{array}{r}-0.27 \\
-0.14 \\
-0.12 \\
-0.20 \\
\\
-0.39 \\
-0.12 \\
-0.18 \\
0.25 \\
0.29\end{array}$ & $\begin{array}{l}\text { T91 } \\
\text { McW90 } \\
\text { McW90 } \\
\text { C\&92 } \\
\text { McW90 } \\
\text { McW90 } \\
\text { McW90 } \\
\text { Z91 } \\
\text { C\&92 }\end{array}$ \\
\hline * & $\begin{array}{l}\text { BS } 4133 \\
\text { US } 3459 \\
\text { BS } 2764 \\
\text { BS } 1112 \\
\text { BS } 5645 \\
\text { BS } 3170 \\
\text { BS } 3364\end{array}$ & \begin{tabular}{|l} 
B1Ib \\
G1I \\
K3I \\
K4Ib \\
K4I \\
M1Ib \\
M2I
\end{tabular} & $\begin{array}{l}\text { CFHT } \\
\text { CFHT } \\
\text { CFHT } \\
\text { CFHT } \\
\text { NTT } \\
\text { GFHT } \\
\text { CFHT }\end{array}$ & \begin{tabular}{|l|}
1 ISIS/IR \\
ISIS/IR \\
ISIS/IR \\
ISIS/IR \\
IRSPEC2 \\
ISIS/IR \\
ISIS/IR.
\end{tabular} & $\begin{array}{r}-0.11 \\
0.15\end{array}$ & $\begin{array}{l}C \& 292 \\
\operatorname{C\& g} 92\end{array}$ \\
\hline
\end{tabular}

Note to Table 1:

BG90 : Barbuy \& Grenon, 1990

C\&92 : Cayrel et al., 1992

McW90: McWilliam, 1990

T91 : Taylor, 1991

Z91 : Zhou, 1991
Table 2. Line identification

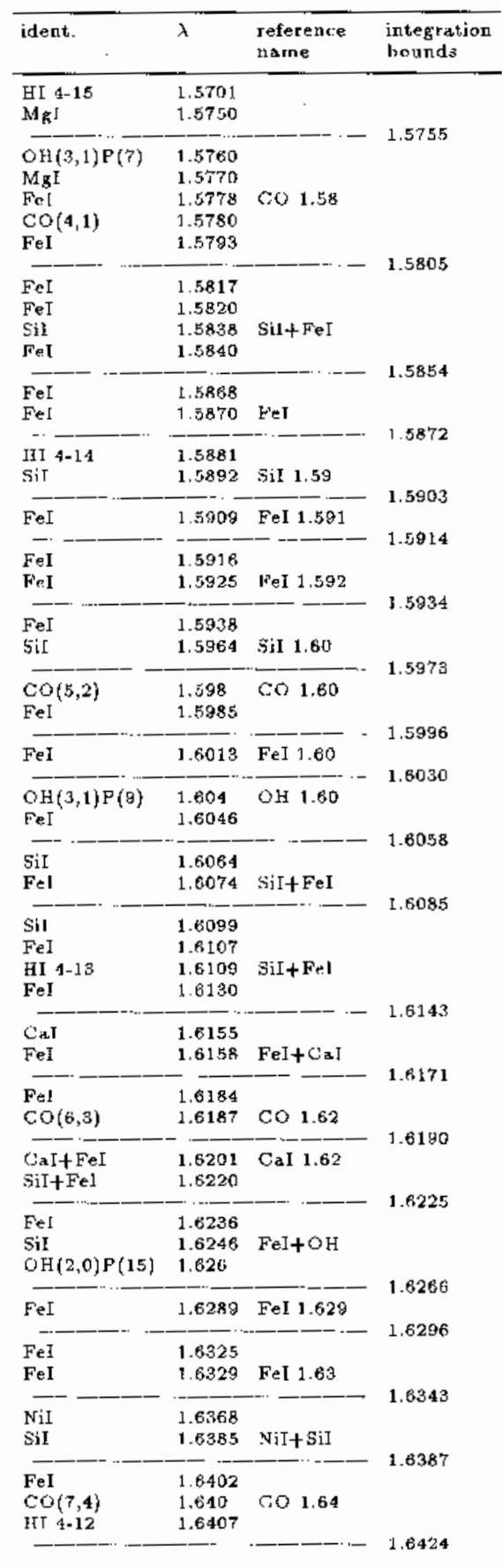

\section{The ISIS/IR spectrograph}

ISIS/IR is a fibre-fed, 'warm' grating spectrograph, working in the $J$ and $H$ bands $(1.0 \mu \mathrm{m} \leq \lambda \leq 1.8 \mu \mathrm{m})$. It is a field spectrograph, i.e. it is possible to record spectra of contiguous points in a small field of view (up to $12^{\prime \prime}$ diameter on the sky). It is now designed to work with one of the Canada-France-Hawaii Telescope (CFHT) RedEye camera (Simons 1993). The spectrograph is mounted on 

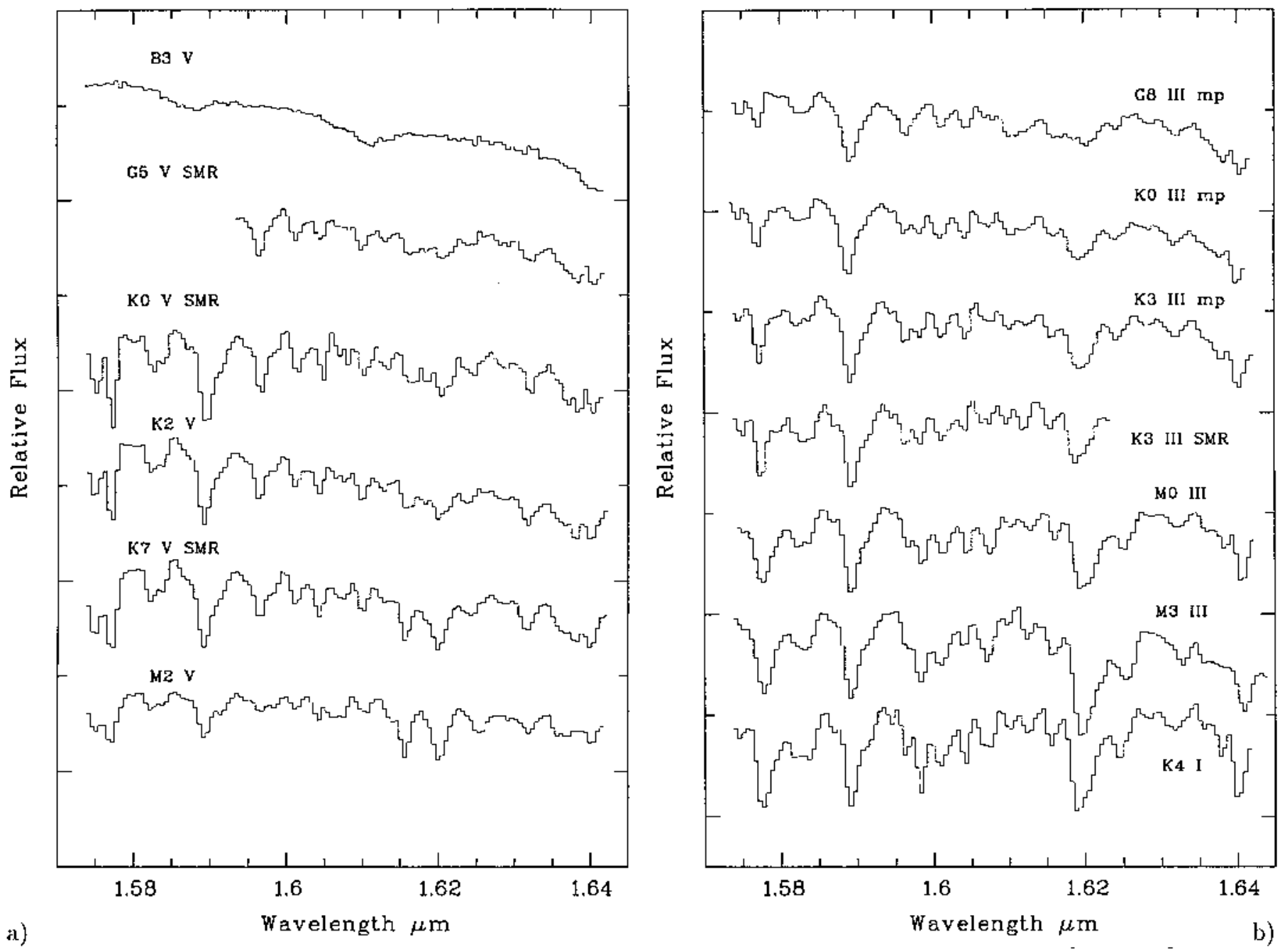

Fig. 1. a-b) The IRSPEC sample, resolution $R=1500$. Spectra are plotted in relative fluxes normalized to 1 . in the range $1.59290-1.59506 \mu \mathrm{m}$. In Fig. 1a, the shift between two spectra is 1.25 , in Fig. $1 \mathrm{~b}$, it is 1.0

an optical bench, and coupled to the telescope with a fibre bundle. It is set on the dome floor, independent from the telescope motions. Following the light path, one can find:

- the so-called 'fibre-environment', attached to the Cassegrain focal plane. In a general way, this device provides 3 functions: focal reduction, guiding and calibration (tungsten and argon sources for flat-field and spectral calibration). At the CFHT, the MOS-SIS bonnette serves as a fibre environment, fulfilling all the required functions.

- the fibre bundle. 'Dry-silica' fibres are used, providing more than $90 \%$ of internal transmission in $J$ and $H$ bands over a 15 meter length. 51 fibres sample the object at the focal plane, while 10 surrounding fibres simultaneously record the sky around the object. Fibre to fibre sampling is adjustable, with a focal reducer, from 0.7 to $1.2^{\prime \prime}$ per fibre. Fibres are aligned at the spectrograph side, forming a pseudo-slit that covers the 256 pixels height of the NICMOS 3 chip. This configuration allows to sample a little extended object and thus offers the possibility of spectro-imaging.

- the spectrograph itself. The design is based on a classical Ebert-Fastie configuration with a parabolic mirror, which provides a good image quality over the whole detector. Two resolving powers are currently available (around 400 and 2000 in both $J$ and $H$ ).

For a resolving power of $R=400$ and one hour exposure ( 1 arcsec object, $10 \sigma$ detection), the limiting magnitude is $m_{J}=17$ to 18 in the $J$ band ( 5 to $210^{-16} \mathrm{~W} / \mathrm{m}^{2} / \mu \mathrm{m}$ ), and $m_{H}=16$ to 17 in $H$ (about the same flux). For $R=2000, m_{J}=15$ to 16 (30 to $\left.1210^{-16} \mathrm{~W} / \mathrm{m}^{2} / \mu \mathrm{m}\right)$, $m_{H}=15\left(1210^{-16} \mathrm{~W} / \mathrm{m}^{2} / \mu \mathrm{m}\right)$. The performances and 

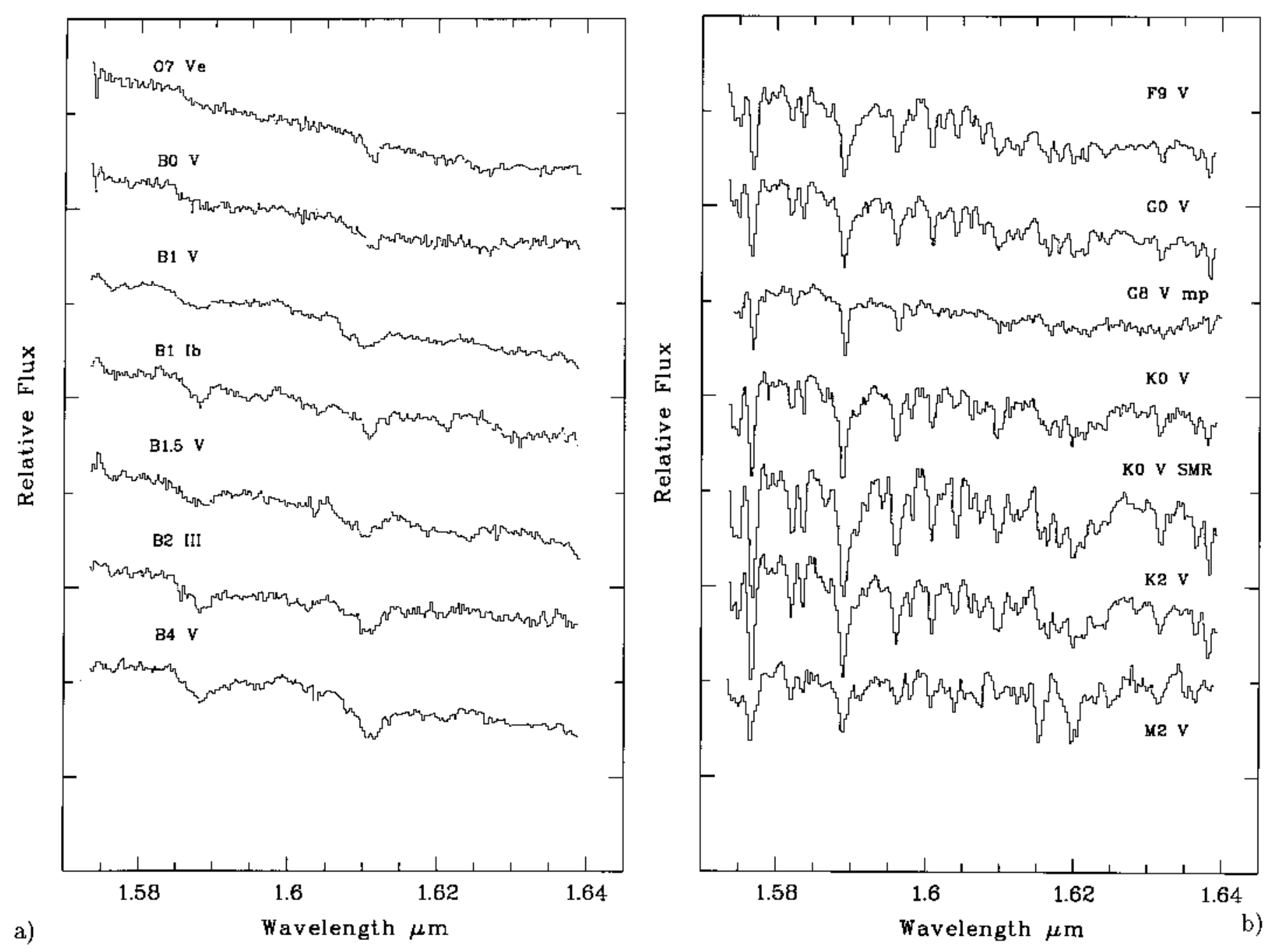

Fig. 2. a-d) The ISIS sample, resolution $R=2000$. Spectra are plotted in relative fluxes normalized to 1 . in the range 1.59290-1.59506 $\mu \mathrm{m}$. In Fig. 2a-c the shift between two spectra is 1.0, in Fig. 2d, it is 1.5

design of ISIS/IR are described in details in Dallier (1995).

\section{Observations}

During runs mainly dedicated to the observation of central parts of active galactic nuclei, two samples of stars have been observed under the same conditions as the galactic nuclei. A sample of 12 stars has been observed with IRSPEC at the ESO-NTT telescope in april 1992. The detector is a $58 \times 62$ SBRC pixel array. Grating 2 at order 1 (see Gredel \& Moorwood 1991) and a 2-pixel wide slit give a spectral resolution $R \simeq 1500$ at $1.60 \mu \mathrm{m}$. For each star we obtained spectra at 3 different grating positions, with an overlap of 30 pixels, to cover the full wavelength range of interest.

The second sample contains 30 stars observed with ISIS/IR (see Sect. 2) at the CFHT in january 1994. The focal reducer used gives a sampling of $1.2^{\prime \prime}$ per fibre; each fibre projects onto about 3 NICMOS pixels. With the 300 lines/mm grating a resolution $R \simeq 2000$ is achieved. The wavelength domain is covered in one ISIS observation.

In Table 1 are given a list of the stars with their spectral type and metallicity $([\mathrm{Fe} / \mathrm{H}])$ when it is known, together with the telescope and spectrograph used. Metallicities are taken from Cayrel de Strobel et al. (1991), unless a reference is quoted in the last column.

The NTT data have been reduced using the IRSPEC context in the ESO-MIDAS package. Each frame was dark subtracted and flat-fielded using an internal halogen lamp at same wavelength and similar counts level. Observations of the stars were made with the source at two different slit positions (typical offset along the slit was of $30^{\prime \prime}$ ) for subsequent integrations. First order sky subtraction was then easy. In case of poor cancelation of sky emission (that happens for some of the least bright stars or when weather 

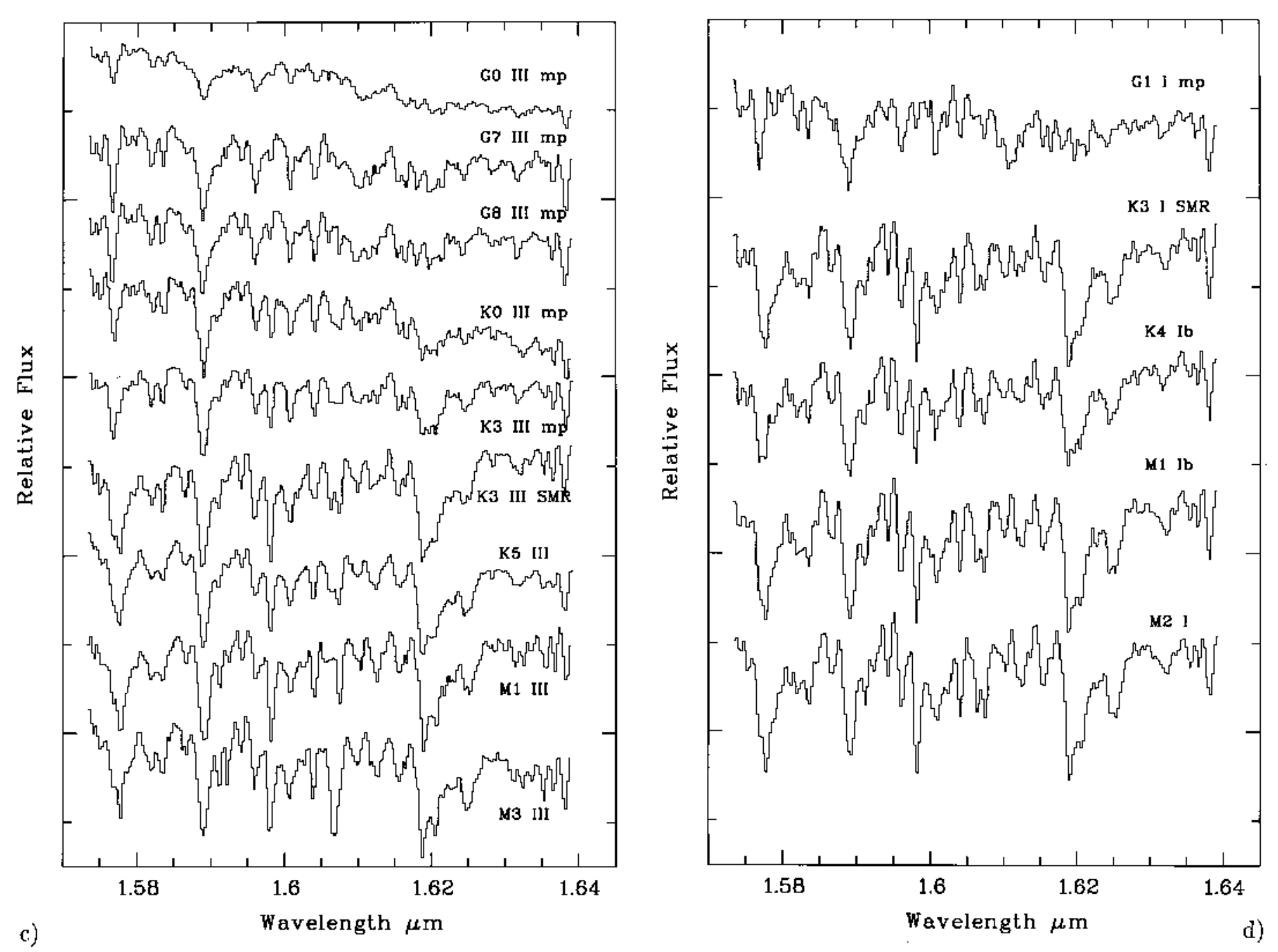

Fig. 2. continued

conditions were poor), second order correction was made by extracting a sky 1D spectrum from part of the slit away from the star. We relied on the 'master curve' for wavelength to grating position conversion provided at the beginning of the run. This was verified at beginning and middle of the run to always reproduce within half a pixel. One-dimension spectra have been extracted summing all relevant cross-sections on the two-dimension spectra.

For ISIS data, a combination of IRAF routines and ESO-MIDAS ones was used. Here also each frame was dark subtracted and flat-fielded using an external halogen lamp. Flats were also used to locate individual fibres on the chip $(x-y$ position and projected width). For each frame, a 1D spectrum from each fibre is then extracted. Spectra were wavelength calibrated using $\mathrm{OH}$ sky lines (see Oliva \& Origlia 1992 for a table). The achieved precision is better than $1 / 7$ pixel. Sky subtraction was then performed using the so-called 'sky fibres' as no dedicated background exposures were taken. The main problem was that each fibre gives a slightly different spectral resolution, enough to produce P-Cygni profiles. We thus had to degrade all individual spectra (sky and object fibres) to the lowest resolution. Final stellar spectra were obtained by summing fibre 1D spectra with signal.

Relative flux calibration was performed using stars obtained during the same night as the objects of interest. During both runs primary flux standards from the lists in Koornneef (1983) and Bouchet et al. (1991) were observed. However, apart from BS4638, they are cool strong lined stars. So, hot stars with good published photometry (Gezari et al. 1993) were used as secondary standards. An asterisk in Table 1 marks all stars finally used as 'standards'. Black body were fitted to the published broad band magnitudes. It was verified that computed black body temperatures are in reasonable agreement with those expected from the star spectral type. Resulting 
normalized flux calibration curves compare to a $3 \%$ level for the NTT data and to a $7 \%$ level for the ISIS one. Thanks to the relative flatness of cool star energy distribution in the small wavelength range considered, it appeared that all primary standards were suitable for relative flux calibration in spite of strong molecular band absorptions. Night averaged curves were used to calibrate both samples in relative flux. For a given star, the individual rectified spectra in the 3 wavelength ranges of IRSPEC data have different energy levels due to the use of an averaged calibration curve and to variable sky conditions. However in the overlapping segments, the spectral shapes agree very well, the individual spectra thus smoothly merged. Fluxes of the final spectra have been normalized to 1 in the range $1.59290-1.59506 \mu \mathrm{m}$. A cross check of the quality of the relative flux calibration can be made by comparing stars observed with both spectrographs (see below).

Special care has been taken to correct from the atmospheric absorption features. BS 2456 (O7) and BS 6187 (O5) were frequently observed for that purpose at $\mathrm{CFH}$ and ESO respectively. These stars are featureless at the given resolution and in the considered wavelength range.

The wavelength range covers by the two sets of observations are very similar: it is $1.573 \mu \mathrm{m}-1.646 \mu \mathrm{m}$ at NTT and $1.578 \mu \mathrm{m}-1.642 \mu \mathrm{m}$ at CFHT. The main features in this range are the $\mathrm{CO}$ overtones $(\Delta v=3)$ at $1.578,1.598,1.619$ and $1.640 \mu \mathrm{m}$, and the $\mathrm{OH}$ absorption bands at 1.576, 1.604, 1.625 and $1.645 \mu \mathrm{m}$. A great number of metallic lines are also present in our stellar spectra. Among them is the strong SiI line at $1.589 \mu \mathrm{m}$. A detailled identification of all the lines has been done using the solar spectrum published by Livingston \& Wallace (1991). Table 2 gives the line identifications with their wavelength. The wavelength range of integration used to measure the equivalent width (EW) is also given (last column).

\section{Results}

Representative spectra of stars from $\mathrm{O}$ to $\mathrm{M}$ for luminosity classes I, III and V are shown in Figs. 1 and 2 for the IRSPEC and ISIS sample respectively. Spectra are plotted in relative fluxes $f_{\lambda}$ normalized to 1 in the range 1.59290-1.59506 $\mu \mathrm{m}$. Evolution of the depth of the absorption lines with spectral type is immediately apparent.

Figure 3 shows the spectra of HD 93800 obtained with both spectrographs. The similarity is remarkable. Note that the better spectral resolution of ISIS/IR allows to separate the numerous weak metallic lines present in cool stars. The difference in resolution explains the different depth (and width) of the lines. It will have consequences on the equivalent width values (see below).

In Table 3 are given the equivalent widths (in $\AA$ ) of all the features for the whole sample of stars. A global continuum has been applied for each star, which accounts for the general shape of the spectrum. Features are labelled with

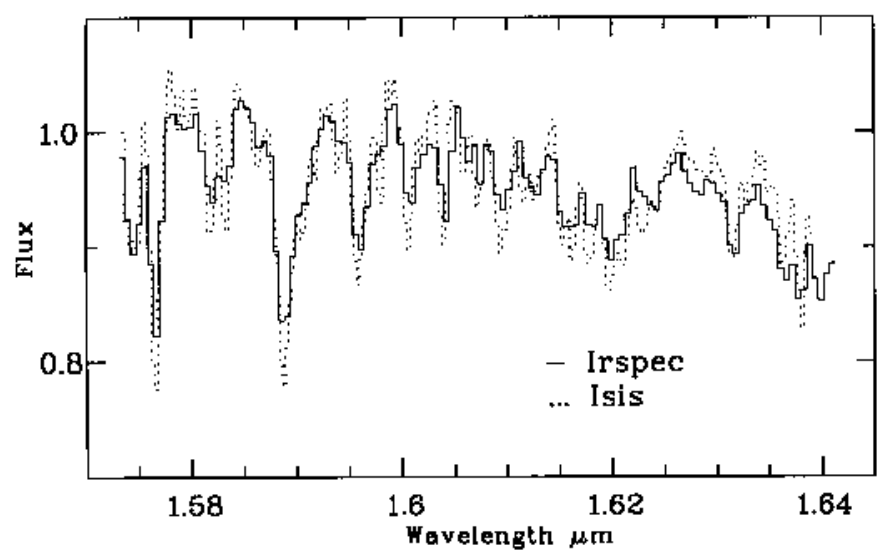

Fig. 3. Spectra of HD 93800 obtained with the IRSPEC and ISIS spectrographs. The difference in resolution explains the different depth of the lines. The better spectral resolution of ISIS/IR allows to separate the numerous weak metallic lines present in cool stars

the identification of the main line responsible for them. In fact, the feature labelled $\mathrm{CO} \lambda 1.58 \mu$ should be called MgI $\lambda 1.577 \mu$ for dwarf stars as it is the dominant line (see Figs. 1 and 2). The wavelength range of integration is also given for the few hot stars as they are different from that of the cool stars (Table 2). Whenever stellar spectra of stars of the same spectral type are similar, they have been averaged. It is the case of two stellar spectra, BS5824 and BS6973 (K3III) in the IRSPEC sample, and of two others, BS2731 and HD 109655 (K5III) in the ISIS one. The listed equivalent widths are those of the averaged spectrum.

Comparing individual stars of same spectral type and/or stars observed more than once in the same configuration, the larger discrepancies are $\Delta \mathrm{EW}=0.1$ and $\Delta \mathrm{EW}=0.3 \AA$ for the strong and weak lines respectively. These are 'measurement' errors but the main source of uncertainty is really the assessment of the continuum level and shape. What appeared to be reasonable minimum and maximum levels of the continuum lead to at most $20 \%$ uncertainty on the measured EW.

The decrease of resolution between ISIS and IRSPEC precludes direct comparison. Indeed the effect of broadening due to lower resolution, is to decrease the measured equivalent widths. That the discrepancy between the two measurements (cf. Table 3) of the five stars in common in the IRSPEC and ISIS/IR samples (HD 93800, GL382, BS5020, BS4695, BS4902) is due to that problem was checked by degrading the ISIS/IR spectral resolution and remeasuring. Broadening correction curves at various spectral resolutions can in principal be constructed by measuring the EW after convolution of the initial spectrum with gaussians of different widths. However, this works only for isolated lines, the factor varying for line blends depending on the number and strength of the 
Table 3. Equivalent widths in $\AA$

\begin{tabular}{|c|c|c|c|c|c|c|c|c|c|c|c|c|c|c|c|c|c|c|c|}
\hline \multicolumn{20}{|c|}{ IEIS/IR DATA } \\
\hline & \multicolumn{2}{|c|}{$\begin{array}{c}B r 14+H e J \\
1.5855-1.59+5\end{array}$} & \multicolumn{2}{|c|}{$\begin{array}{l}\text { Hel } \\
62-1.6090\end{array}$} & \multicolumn{2}{|c|}{$\begin{array}{c}\text { Frr13 } \\
1.6090-1.6140\end{array}$} & & & & & & & & & & & & & \\
\hline $\begin{array}{l}\text { BS2456 } \\
\text { o }\end{array}$ & \multicolumn{2}{|c|}{0.6} & & & \multicolumn{2}{|c|}{0.8} & & & & & & & & & & & & & \\
\hline $\begin{array}{l}\text { AS1879 } \\
\text { O8 }\end{array}$ & \multicolumn{2}{|c|}{1.6} & \multicolumn{2}{|c|}{0.2} & \multicolumn{2}{|c|}{1.4} & & & & & & & & & & & & & \\
\hline $\begin{array}{l}\text { BS112: } \\
\text { BOV }\end{array}$ & \multicolumn{2}{|c|}{1.7} & \multicolumn{2}{|c|}{0.4} & 1 & 8 & & & & & & & & & & & & & \\
\hline $\begin{array}{l}\text { BS5056 } \\
\text { B1V }\end{array}$ & \multicolumn{2}{|c|}{1.7} & \multicolumn{2}{|c|}{1.0} & \multicolumn{2}{|c|}{2.4} & & & & & & & & & & & & & \\
\hline $\begin{array}{l}\text { BS } 4590 \\
\text { B1.5V }\end{array}$ & \multicolumn{2}{|c|}{2.1} & & .8 & 1 & & & & & & & & & & & & & & \\
\hline $\begin{array}{l}\text { BS41.56 } \\
\text { P34V }\end{array}$ & & .0 & & & 3 & & & & & & & & & & & & & & \\
\hline $\begin{array}{l}\text { BSI790 } \\
\text { B2III }\end{array}$ & & 6 & & 1.7 & 2 & & & & & & & & & & & & & & \\
\hline $\begin{array}{l}\text { OS4133 } \\
B 1 \mathrm{Ib}^{2}\end{array}$ & & .7 & & .7 & 2 & & & & & & & & & & & & & & \\
\hline & $\begin{array}{l}\text { co } \\
1.58\end{array}$ & $\begin{array}{c}\mathrm{SiI} \\
+\mathrm{FeI}\end{array}$ & $\begin{array}{r}\mathrm{FeI} \\
1.58\end{array}$ & $\begin{array}{c}\mathrm{SiI} \\
\mathbf{1 . 5 9}\end{array}$ & $\begin{array}{c}\mathrm{FeI} \\
1.591\end{array}$ & $\begin{array}{c}\mathrm{FeI} \\
1.592\end{array}$ & $\begin{array}{c}\text { SiI } \\
1.60\end{array}$ & $\begin{array}{l}\text { CO } \\
1.60\end{array}$ & $\begin{array}{l}\mathrm{FeI} \\
1.60\end{array}$ & $\begin{array}{l}\mathrm{OH} \\
1.60\end{array}$ & $\begin{array}{l}\mathrm{Sil} \\
+\mathrm{FeI}\end{array}$ & $\begin{array}{c}\text { Sil } \\
+ \text { FeI }\end{array}$ & $\begin{array}{l}\text { Fel } \\
+\mathrm{CaI}\end{array}$ & $\begin{array}{l}00 \\
1.82\end{array}$ & $\begin{array}{l}\mathrm{CaI} \\
\mathrm{l} .82\end{array}$ & $\begin{array}{l}\mathrm{FeJ} \\
+\mathrm{OH}\end{array}$ & $\begin{array}{c}F_{t: \mathbf{I}} \\
+1.829\end{array}$ & $\begin{array}{l}\text { FeT } \\
1.63\end{array}$ & $\begin{array}{l}\mathrm{NiI} \\
+\mathrm{SiI}\end{array}$ \\
\hline $\begin{array}{l}\text { As } 788 \\
\text { F9V }\end{array}$ & 2.2 & 1.6 & 0.7 & 3.1 & 0.7 & 0.7 & 2.3 & 0.5 & 1.5 & 0.7 & 1.0 & 3.6 & 1.6 & 1.8 & 1.5 & 1.6 & 0.8 & 0.9 & 1.1 \\
\hline $\begin{array}{l}\mathrm{BS} 4983 \\
\text { GoV }\end{array}$ & 1.8 & 1.3 & 0.6 & 2.8 & 0.6 & 0.6 & 1.9 & 0.4 & 1.4 & 0.9 & 0.9 & 3.0 & 1.4 & 1.5 & 1.5 & 1.1 & 0.8 & 1.3 & 1.4 \\
\hline $\begin{array}{l}\text { BS4550 } \\
\text { G8V } \mathrm{mp}\end{array}$ & 1.6 & 0.5 & 0.2 & 1.6 & 0.3 & 0.4 & 1.2 & 0.4 & 0.5 & 0.6 & 0.4 & 1.5 & 0.6 & 0.7 & 0.8 & 0.4 & 0.4 & $0.8:$ & $0.1:$ \\
\hline $\begin{array}{l}\text { HD 112758 } \\
\text { KOV }\end{array}$ & 2.7 & 1.3 & 0.7 & 3.3 & 0.7 & 0.5 & 2.1 & 0.6 & 1.1 & 0.6 & 1.1 & 2.2 & 1.4 & 1.8 & 1.8 & 1.4 & 0.4 & I. 1 & 1.1 \\
\hline $\begin{array}{l}\text { HD } 93800 \\
\text { KOV SMR }\end{array}$ & 3.4 & 2.6 & 0.8 & 4.4 & 1.3 & 0.9 & 3.1 & 0.9 & 1.9 & 1.2 & 1.5 & 3.7 & 2.1 & 2.7 & 2.6 & 1.8 & 0.4 & 1.5 & 2.5 \\
\hline $\begin{array}{l}\mathrm{HD115404} \\
\mathrm{K} 2 \mathrm{~V}\end{array}$ & 3.3 & 2.2 & 0.8 & 4.0 & 0.9 & 0.9 & 3.0 & 1.0 & 1.8 & 1.1 & 1.6 & 3.1 & 1.9 & 2.4 & 2.1 & 1.6 & 0.3 & 1.1 & 1.6 \\
\hline $\begin{array}{l}\mathrm{Gl382} \\
\mathrm{M} 2 \mathrm{~V}\end{array}$ & 3.0 & 1.6 & 0.6 & 2.5 & 0.6 & 0.9 & 1.8 & 0.8 & 1.5 & 1.6 & 1.5 & 2.8 & 2.3 & 2.1 & 2.3 & 2.3 & 0.7 & 1.7 & 1.8 \\
\hline $\begin{array}{l}\text { BS4883 } \\
\text { GOIII mp }\end{array}$ & 1.3 & 1.1 & 0.6 & 2.2 & 0.6 & 0.6 & 1.7 & 0.5 & 0.8 & 0.6 & 0.5 & 2.0 & 0.9 & 1.1 & 1.2 & 1.1 & 0.7 & 1.0 & 0.7 \\
\hline $\begin{array}{l}\text { HS3903 } \\
\text { G7III mp }\end{array}$ & 2.6 & 1.7 & 0.7 & 3.6 & 0.9 & 0.7 & 2.4 & 0.7 & 1.9 & 1.2 & 1.3 & 4.3 & 1.9 & 2.2 & 2.1 & 1.9 & 1.2 & 1.8 & 1.8 \\
\hline $\begin{array}{l}\text { BS5020 } \\
\text { G8III mp }\end{array}$ & 2.8 & 2.1 & 0.7 & 3.7 & 0.8 & 0.6 & 2.1 & 0.5 & 1.8 & 1.1 & 1.3 & 3.8 & I. 6 & 2.0 & 1.7 & 1.5 & 0.8 & 1.3 & 1.7 \\
\hline $\begin{array}{l}\text { BS4695 } \\
\text { KoIII mp }\end{array}$ & 2.7 & 1.9 & 0.4 & 3.5 & 0.9 & 0.5 & 1.4 & 1.2 & 1.6 & 0.8 & 1.4 & 2.4 & 1.5 & 2.3 & 2.0 & 2.0 & 0.8 & 2.1 & 2.2 \\
\hline $\begin{array}{l}\text { BS5315 } \\
\text { K3III thp }\end{array}$ & 3.4 & 1.9 & 0.5 & 3.5 & 0.8 & 0.4 & 1.7 & 1.4 & 2.0 & 1.2 & 1.6 & 2.7 & 1.4 & 2.7 & 2.2 & 2.1 & 0.7 & 1.2 & 1.8 \\
\hline $\begin{array}{l}\text { HD111180 } \\
\text { K3III SMR }\end{array}$ & 6.3 & 3.6 & 1.1 & 4.6 & 1.2 & 0.9 & 2.6 & 3.0 & 3.4 & 1.6 & 2.4 & 2.9 & 1.6 & 4.4 & 3.4 & 3.4 & 0.3 & 1.0 & 1.3 \\
\hline $\begin{array}{l}\text { HD109655 } \\
\text { BS2731, K5III }\end{array}$ & 4.6 & 2.4 & 0.6 & 4.1 & 1.0 & 0.9 & 2.2 & 2.7 & 2.8 & 1.6 & 2.1 & 2.2 & 1.1 & 3.8 & 3.3 & 3.4 & 0.5 & 1.1 & 1.6 \\
\hline $\begin{array}{l}\text { BS4127 } \\
\text { M1III }\end{array}$ & 5.8 & 3.2 & 0.6 & 4.2 & 1.0 & 0.9 & 1.8 & 2.8 & 3.0 & 1.8 & 2.2 & 2.6 & 1.3 & 3.8 & 3.8 & 4.0 & 0.8 & 1.8 & 1.8 \\
\hline $\begin{array}{l}\text { BS4902 } \\
\text { M3HI }\end{array}$ & 5.5 & 3.6 & 0.9 & 4.7 & 1.4 & 1.4 & 2.6 & 3.4 & 3.8 & 2.2 & 3.9 & 2.9 & 1.7 & 4.3 & 3.8 & 4.3 & 0.8 & 2.5 & 2.6 \\
\hline $\begin{array}{l}\text { BS3459 } \\
\text { G1I mp }\end{array}$ & 2.7 & 2.1 & 1.1 & 4.5 & 1.1 & 1.0 & 2.4 & 0.8 & 2.0 & 0.9 & 1.4 & 5.2 & 1.9 & 2.2 & 2.1 & 2.5 & 1.2 & 1.5 & 1.7 \\
\hline $\begin{array}{l}\text { BS2764 } \\
\text { K31 SMR }\end{array}$ & 7.4 & 4.5 & 1.0 & 4.8 & 1.4 & 1.1 & 2.7 & 3.8 & 4.7 & 2.1 & 2.8 & 4. 1 & 2.0 & 5.0 & 4.5 & 5.1 & 1.8 & 2.7 & 2.4 \\
\hline $\begin{array}{l}\text { BSi112 } \\
\text { KaIb }\end{array}$ & 7.2 & 4.6 & 1.3 & 5.3 & 1.5 & 1.4 & 3.4 & 3.0 & 4.2 & 2.1 & 2.8 & 4.3 & 2.3 & 4.5 & 3.7 & 4.3 & 1.4 & 1.9 & 2.1 \\
\hline $\begin{array}{l}\text { BS3170 } \\
\text { MiIb }\end{array}$ & 8.5 & 5.6 & 1.4 & 5.5 & 1.7 & 1.7 & 3.3 & 3.9 & 5.1 & 2.5 & 3.5 & 4.4 & 2.4 & 5.1 & 4.5 & 5.3 & 1.3 & 2.6 & 2.3 \\
\hline $\begin{array}{l}\mathrm{B} 53384 \\
\mathrm{M} 2 \mathrm{I}\end{array}$ & 9.4 & 5.8 & 1.3 & 5.6 & 1.6 & 1.7 & 2.8 & 4.2 & 5.6 & 2.8 & 3.9 & 4.3 & 2.2 & 5.3 & 5.0 & 5.8 & 1.5 & 2.8 & $2 . \breve{5}$ \\
\hline & & & & & & & IRSP & EC D & $\mathrm{ATA}$ & & & & & & & & & & \\
\hline & $\begin{array}{c}C O \\
1.58\end{array}$ & $\begin{array}{c}\mathrm{SiI} \\
+\mathrm{FeI}\end{array}$ & $\begin{array}{c}\text { FeI } \\
1.58\end{array}$ & $\begin{array}{c}\text { SiI } \\
1.59\end{array}$ & $\begin{array}{c}\text { Fel } \\
1.591\end{array}$ & $\begin{array}{c}\text { FeI } \\
1.592\end{array}$ & $\begin{array}{c}5 i 1 \\
1.80\end{array}$ & $\begin{array}{l}\mathrm{co} \\
1.60\end{array}$ & $\begin{array}{r}\mathrm{FeI} \\
1.60\end{array}$ & $\begin{array}{c}\mathrm{OH} \\
1.60\end{array}$ & $\begin{array}{c}\mathrm{SiI} \\
+\mathrm{FeI}\end{array}$ & $\begin{array}{c}\text { SiI } \\
+\mathrm{FeI}\end{array}$ & $\begin{array}{c}\mathrm{FeI} \\
+\mathrm{Cal}\end{array}$ & $\begin{array}{l}00 \\
1.62\end{array}$ & $\begin{array}{l}C_{2}[ \\
1.62\end{array}$ & $\begin{array}{c}\text { FeI } \\
+O H\end{array}$ & $\begin{array}{c}\mathrm{FeI} \\
+1.629\end{array}$ & $\begin{array}{l}\text { FeI } \\
1.63\end{array}$ & $\begin{array}{c}\mathrm{NiI} \\
+\mathrm{SiI}\end{array}$ \\
\hline $\begin{array}{l}\text { HDl60691 } \\
\text { G5V SMR }\end{array}$ & & & & & & & 2.4 & 0.6 & 1.2 & 1.0 & 0.6 & 2.2 & 1.3 & 1.4 & 1.2 & 0.5 & 0.2 & L. 1 & 2.0 \\
\hline $\begin{array}{l}\text { HD } 93800 \\
\text { KOV SMR }\end{array}$ & 3.5 & 2.3 & 0.4 & 3.7 & 1.1 & 0.8 & 2.5 & 0.9 & 1.6 & 1.4 & 1.0 & 2.7 & 1.6 & 1.9 & 1.9 & 1.1 & 0.4 & 1.1 & 2.2 \\
\hline $\begin{array}{l}\mathrm{HD} 130871 \\
\mathrm{~K} 2 \mathrm{~V}\end{array}$ & 3.2 & 2.1 & 0.4 & 3.3 & 0.8 & 0.6 & 2.0 & 0.6 & 1.2 & 1.1 & 0.7 & 2.0 & 1.4 & 1.5 & 1.3 & 0.8 & 0.3 & t. 4 & 2.1 \\
\hline $\begin{array}{l}\text { IID } 157881 \\
\text { K7V SMR }\end{array}$ & 4.0 & 2.1 & 0.4 & 3.5 & 0.9 & 0.6 & 2.0 & 0.7 & 1.1 & 1.3 & 0.8 & 1.8 & 2.1 & 2.1 & 1.8 & 1.1 & 0.2 & 1.5 & 2.3 \\
\hline $\begin{array}{l}\text { G1382 } \\
\text { M2V }\end{array}$ & 2.1 & 0.7 & 0.1 & 1.6 & 0.4 & 0.4 & 0.7 & 0.4 & 0.5 & 0.9 & 1.0 & 1.2 & 1.9 & 1.4 & 1.6 & 1.2 & 0.3 & 0.9 & 1.2 \\
\hline $\begin{array}{l}\mathrm{B} 55020 \\
\text { GSIII }\end{array}$ & 1.1 & 1.1 & 0.2 & 2.8 & 0.7 & 0.4 & 1.6 & 0.6 & 1.1 & 0.8 & 0.6 & 2.6 & 1.2 & 1.6 & 1.4 & 0.8 & 0.3 & 1.1 & 2.1 \\
\hline $\begin{array}{l}\text { BS } 4695 \\
\text { KOIII mp }\end{array}$ & 2.1 & 1.3 & 0.3 & 3.0 & 0.7 & 0.3 & 1.0 & 0.9 & 1.4 & 0.9 & 0.6 & 1.6 & 0.8 & 1.8 & 1.3 & 1.1 & 0.3 & 0.9 & 1.6 \\
\hline $\begin{array}{l}\mathrm{BS5821} \\
\text { BS6973, K3III mp }\end{array}$ & 3.1 & 2.0 & 0.4 & 3.5 & 1.0 & 0.7 & 2.1 & 1.5 & 2.0 & 1.5 & 0.7 & 2.3 & 1.4 & 2.7 & 2.0 & 1.6 & 0.5 & 1.1 & 1.9 \\
\hline $\begin{array}{l}\text { HD175674 } \\
\text { K.IIJ SMR }\end{array}$ & 3.9 & 3.0 & 0.5 & 3.5 & 1.0 & 0.8 & 1.9 & 1.5 & 2.0 & 0.9 & 1.0 & 1.8 & 1.1 & 2.6 & 1.1 & & & & \\
\hline $\begin{array}{l}\text { BS4174 } \\
\text { MOIII }\end{array}$ & 4.5 & 2.5 & 0.3 & 3.2 & 0.9 & 0.5 & 1.2 & 1.9 & 2.3 & 1.6 & 1.8 & 1.7 & 1.0 & 2.7 & 2.8 & 2.6 & 0.4 & I. 1 & 1.9 \\
\hline $\begin{array}{l}\text { BS4902 } \\
\text { M3III }\end{array}$ & 5.0 & 2.8 & 0.4 & 3.6 & 0.9 & 0.8 & 1.4 & 2.6 & 2.8 & 1.3 & 2.0 & 1.4 & 1.6 & 4.0 & 4.0 & 3.6 & 0.1 & 1.2 & 1.6 \\
\hline $\begin{array}{l}\text { B35645 } \\
\text { K4I }\end{array}$ & 5.7 & 3.8 & 0.5 & 3.8 & 1.0 & 0.7 & 1.6 & 2.6 & 2.9 & 1.7 & 1.7 & 1.6 & 1.2 & 3.9 & 3.0 & 2.7 & 0.4 & 1.2 & 2.0 \\
\hline
\end{tabular}


$\mathrm{OH} \quad 1.6 \mu \mathrm{m}$
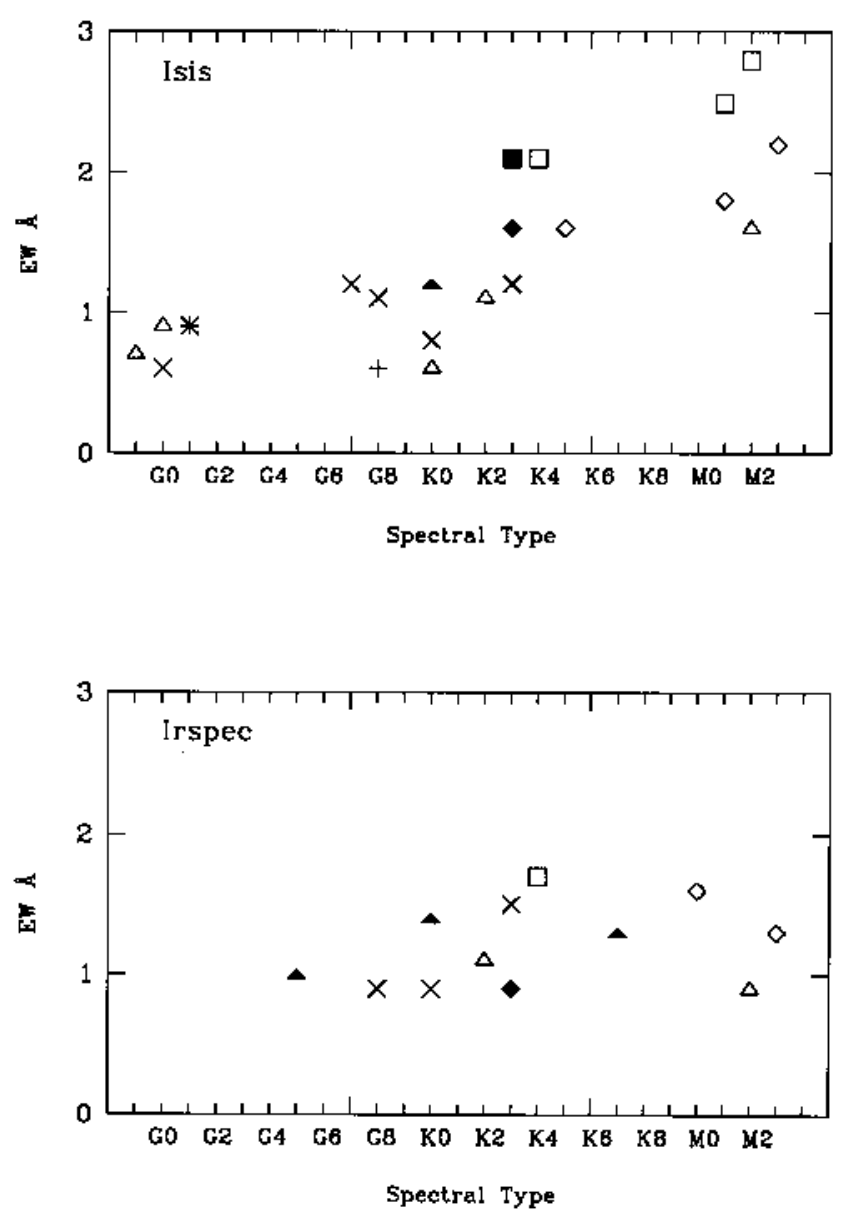

Fig. 4. Variations of $\mathrm{OH} 1.60 \mu \mathrm{m}$ with spectral type and luminosity class. Open symbols are for normal abundance stars, filled symbols are for SMR stars and crosses for underabundant ones. $\square, \boldsymbol{\square}$ and $*$ : supergiants; $\diamond, \diamond$ and $\times$ : giants; $\triangle, \boldsymbol{\Delta}$ and + : dwarfs

included lines. To create a stellar library merging both samples, ISIS spectra have to be degraded to the resolution of the IRSPEC ones. However, for stellar population synthesis of a galaxy observed with the best resolution, only the limited - better resolved - library will be available, except if the galactic velocity dispersion is high. In that case the whole stellar library has in fact to be degraded to the dispersion of the galaxy.

Two stars of our sample (BS5645 and BS3364) are found in Origlia et al. (1993). These authors give the equivalent width of two strong features, SiI $1.59 \mu \mathrm{m}$ and $\mathrm{CO} 1.62 \mu \mathrm{m}$. However they used a local continuum and wider integration bands. To compare with their results, $\mathrm{EW}$ of both lines were remeasured following the prescription as given in their Sect. 2.2. For BS3364, we also had to degrade the resolution to match IRSPEC. For BS5645,
FeI $1.6 \mu \mathrm{m}$
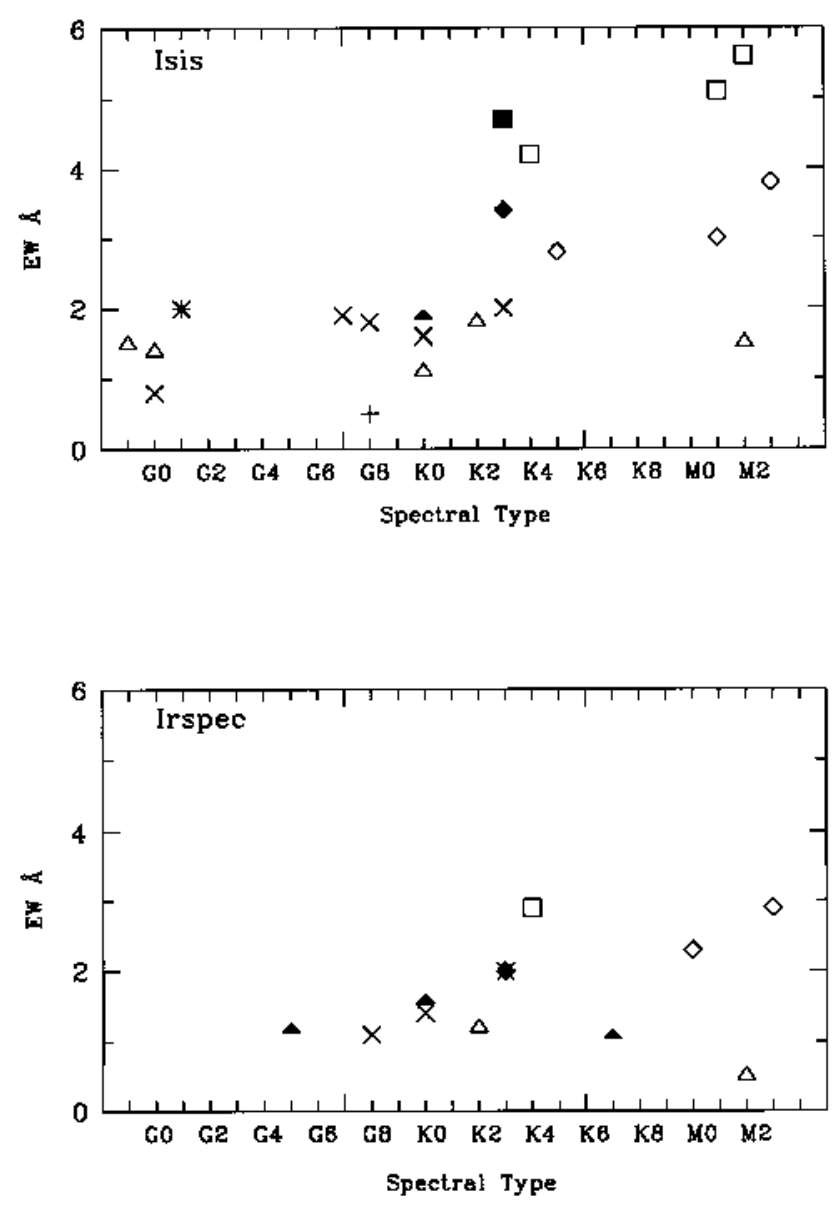

Fig. 5. Variations of FeI $1.60 \mu \mathrm{m}$ with spectral type and luminosity class. Same symbols as in Fig. 4

our new EW are $0.3 \AA$ lower for both lines. In the case of BS3364, they are $0.4 \AA$ larger. This is within the above quoted errors when one also keeps in mind that we had to degrade ISIS resolution. A priori, for stellar population synthesis at a resolution lower or equal to 1500 , their library can be added to the one presented here.

In Figs. 4 to 6 are given the variation of, respectively, $\mathrm{OH} 1.60 \mu, \mathrm{FeI} 1.60 \mu \mathrm{m}$ and $\mathrm{CO} 1.62 \mu \mathrm{m}$ as a function of spectral type and luminosity class, for both sets of data. Normal stars are plotted together with over and under abundant stars, printed with different symbols. In spite of the small number of stars, general tendencies can be inferred. The discussion below applies to both samples although they are presented separately because of the small shift in EW due to the difference in resolution as explained above.

First, all three lines are good indicators of the effective temperature, being stronger in cooler stars. Note how close are the behaviour of the $\mathrm{CO}$ and $\mathrm{Fe}$ features. 
Co $1.62 \mu \mathrm{m}$
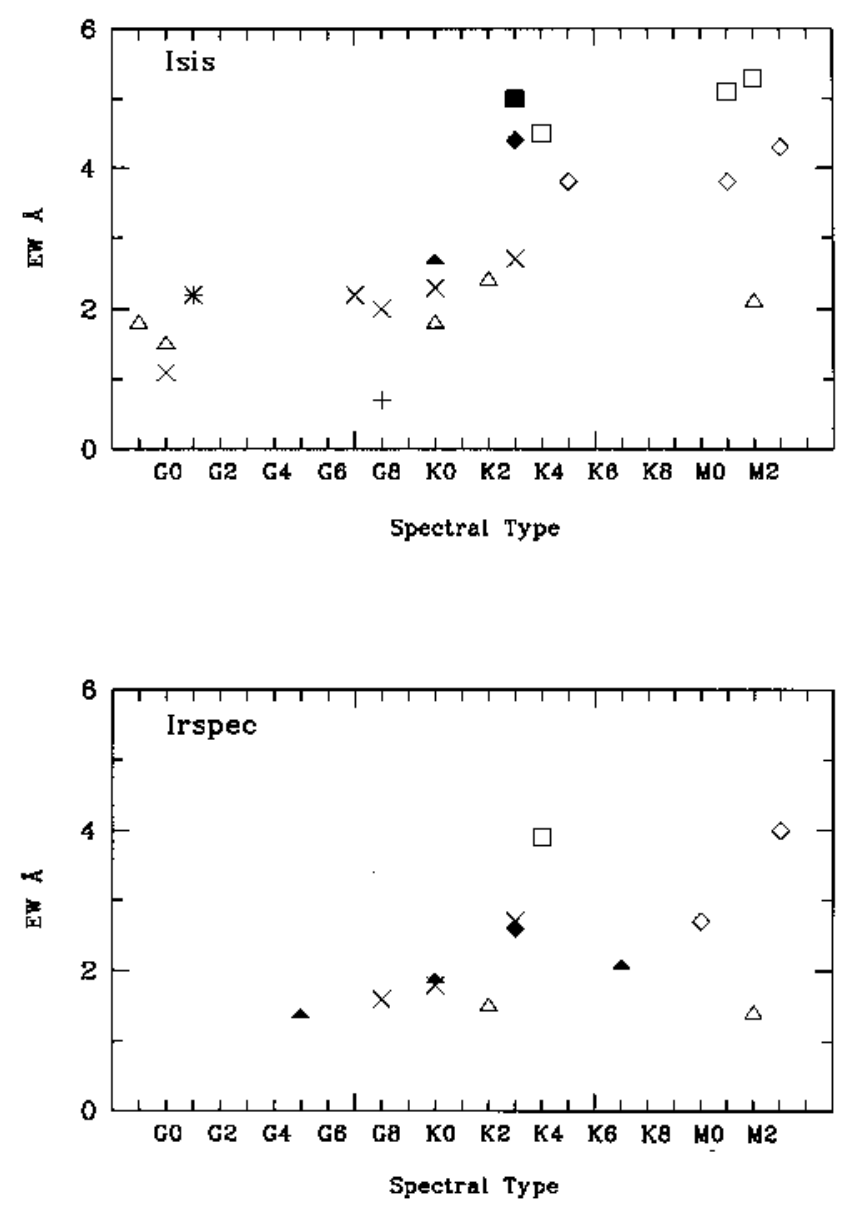

Fig. 6. Variations of CO $1.62 \mu \mathrm{m}$ with spectral type and luminosity class. Same symbols as in Fig. 4

Second, although none of these lines are strong luminosity class indicators, $\mathrm{CO}$ and $\mathrm{FeI}$ discriminate pretty well supergiants from dwarfs and giants. Their equivalent widths are clearly larger than those of the less luminous stars.

Third, it has to be emphasised that stars plotted as normal-abundance stars are actually stars for which abundances are not known. Some of them could have in fact non solar abundances. It could be the case for the dwarf GL $382(\mathrm{M} 2 \mathrm{~V})$ the lines of which are systematically weaker than expected for a M2 star if it follows the general trend of the more luminous stars. GL 382 could then be an underabundant star. However a similar trend appears in the $\mathrm{K}$ and $\mathrm{M}$ dwarf stellar spectra in Lançon \& RoccaVolmerange (1993) where IR stellar features are maximum in $\mathrm{K} 3 \mathrm{~V}$ type and decrease beyond.

We also plot, Fig. 7, the variation of the line ratio $(\mathrm{CO}+\mathrm{CaI}) \lambda 1.62 / \mathrm{SiI} \lambda 1.59$ as a function of spectral type as it extends to stars warmer than the one presented by
Co $1.62 / \operatorname{Sil} 1.59$
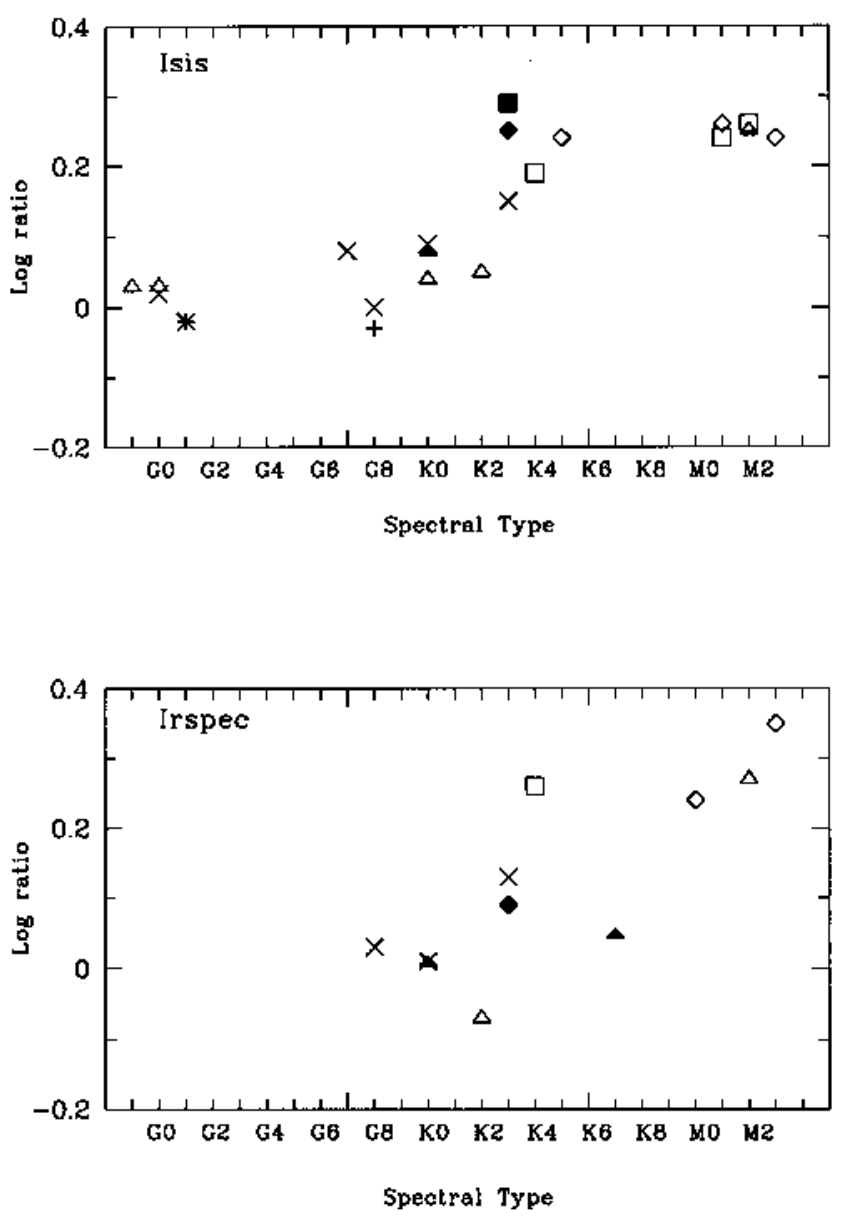

Fig. 7. Variations of the line ratio, $(\mathrm{CO}+\mathrm{CaI}) 1.62 \mu \mathrm{m}$ over SiI $1.59 \mu \mathrm{m}$, with spectral type and luminosity class. Same symbols as in Fig. 4

Origlia et al. (1993). CO+CaI had to be plotted to be comparable to their broad feature. This ratio is actually a very good temperature indicator but it does not allow any discrimination between luminosity classes or metallicity ranges as a consequence of the sensitivity of both lines on luminosity and metallicity. For IRSPEC data, the slope and scatter of the relation is the same as the one obtained by Origlia et al. For the ISIS data, although the scatter is of the same order, the relation is along a different slope due to the presence, here, of G stars.

In Fig. 8 is plotted $\operatorname{CO} \lambda 1.60 / \operatorname{SiI} \lambda 1.60$, a very good luminosity discriminator for stars later than K0. This is indeed obvious by inspection of Figs. 1 and 2. The advantage of this ratio is that both lines are next to each other so that they can be observed at the same time and do not suffer from any reddening problem. 
$\operatorname{CO} 1.6 / \operatorname{SiI} 1.6$
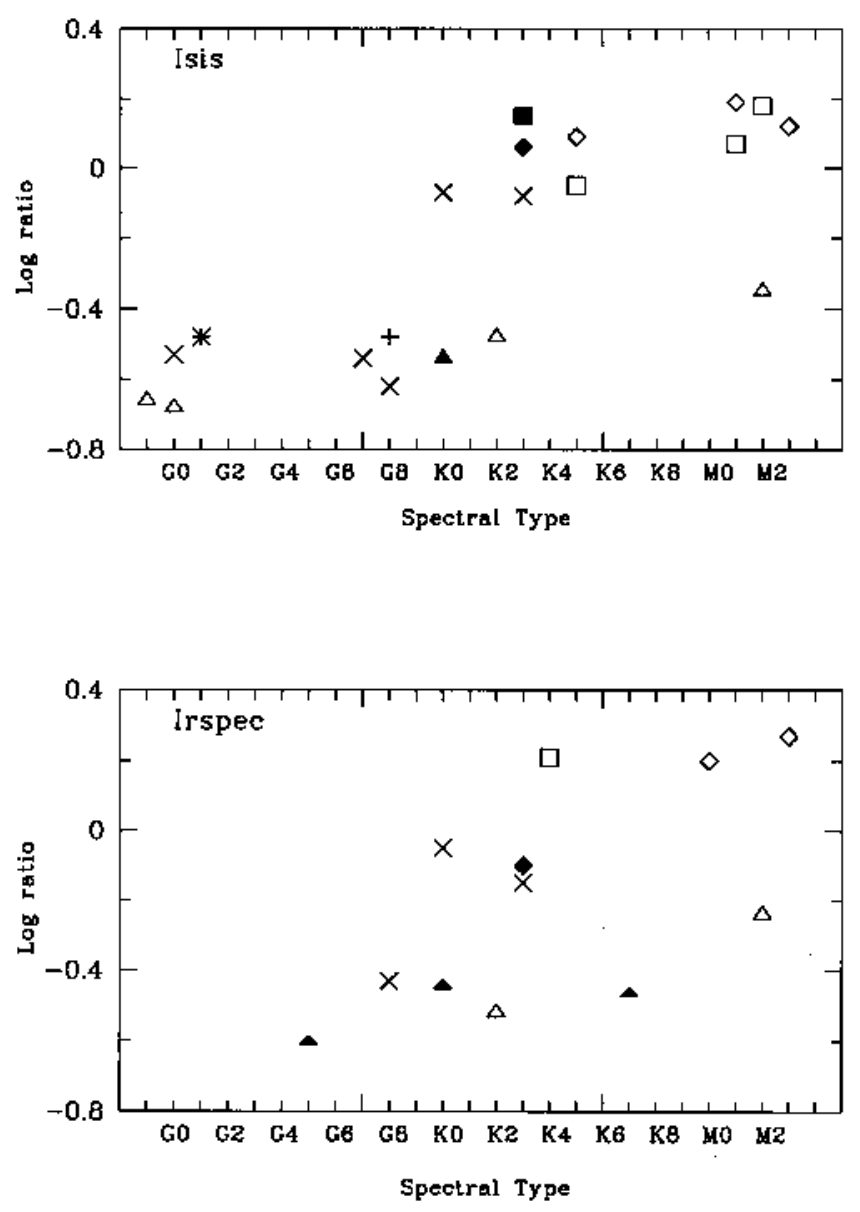

Fig. 8. Variations of the line ratio, CO $1.6 \mu \mathrm{m}$ over SiI $1.6 \mu \mathrm{m}$, with spectral type and luminosity class. Same symbols as in Fig. 4

The spectra, at their full spectral resolution, are accessible in electronic form through the Centre de Données Stellaire in Strasbourg.

Acknowledgements. We wish to thank Jean-Gabriel Cuby and Jean Czarny for their important participation to the ISIS-IR run of January 1994. We are also grateful to Alan Moorwood for useful comments as a referee.

\section{References}

Arnaud K.A., Gilmore G., Collier Cameron A., 1989, MNRAS 237, 495

Barbuy B., Grenon M., 1990, ESO Conf. Proc. 35 "Bulge of galaxies". In: Jarvis B.J. and Terndrup D.M. (eds.), La Serena

Bell R.A., Briley M.M., Smith G.H., 1990, AJ 100, 187

Bouchet P., Manfroid J., Schmider F.-X., 1991, A\&AS 91, 409

Casali M.M., Matthews H.E., 1992, MNRAS 258, 399

Cayrel de Strobel G., 1991, conf. proc. "evolutionary phenomena in the Universe", p. 27

Cayrel de Strobel G., Hauck B., François P., et al., 1992, A\&AS 95, 273

Dallier R., 1995, PhD Thesis, Paris 7 University

Davidge T.J., 1990, AJ 99, 561

Davidge T.J., 1992, AJ 103, 1512

Davidge T.J., Boeshaar P.C., 1993, ApJ Lett. 403, L47

Delisle S., Hardy E., 1992, AJ 103, 711

Gaffney N.I., Lester D.F., 1992, ApJ 394, 139

Gezari D.Y., Schmitz M., Pitts P.S., Mead J.M., 1993, Nasa Ref. Pub. 1294, "Catalog of IR observations", 3rd edition

Gredel R., Moorwood A.F.M., 1991, IRSPEC-ESO operating manual

Kleinmann S.G., Hall D.N.B., 1986, ApJS 62, 501

Koorneef J., 1983, A\&AS 51, 489

Lançon A., Rocca-Volmerange B., 1992 A\&AS 96, 593

Livingston W., Wallace L., 1991, N.S.O. Technical report 91001, National Solar Observatory, Tucson

McWilliam A., 1990, ApJS 74, 1075

Mould J.R., 1978, ApJ 226, 923

Oliva E., Origlia L., 1992, A\&A 254, 466

Origlia L., Moorwood A.F.M., Oliva E., 1993, A\&A 280, 536

Simons D., 1993, The RedEye Camera, 'CFHT user's manual'

Taylor B.J., 1991, ApJS 76, 715

Terndrup D.M., Frogel J.A., Whitford A.E., 1991, ApJ 378, 742

Tsuji T., 1986, A\&A 156, 8

Tsuji T., 1991, A\&A 245, 203

Vila-Costas M.B., Edmunds M.G., 1992, MNRAS 259, 121

Zhou X., 1991, A\&A 248, 367 